\title{
Study of The Effect of Repetitive Transcranial Magnetic Stimulation on A Sample of Children with Autism Spectrum Disorder
}

Ali Ismail Abd alrahman, Amgad Ahmed Moshref Gabr, Tarek Ibrahim Mohamed Elfekey*

Department of Psychiatry, Faculty of Medicine, Al-Azhar University, Cairo, Egypt

Corresponding author: Tarek Ibrahim Mohamed Elfekey, E-mail: tarek.elfekey1991@ gmail.com, Mobile: (+20)01064506788

\section{ABSTRACT}

Background: Autism Spectrum Disorder (ASD) is a behaviorally defined complex neurodevelopmental syndrome. ASD is one of the most common child psychiatric disorders. Despite the long history of research on ASD, no much is known yet about the exact biological causes and how the disorder can be effectively treated.

Objective: To study clinical effect of repetitive Transcranial Magnetic Stimulation (rTMS) on a sample of children with autism spectrum disorder.

Patients and Methods: the sample consisted of 30 children. Their ages ranged from 4 to 10 years old. After being diagnosed clinically according to DSM-5 through a designed semi-structured interview and through application of childhood autistic rating scale CARS and assessment of the degree of clinical severity of autism spectrum disorders according to DSM-5.

Results: The results of the study after the completion of 12 sessions of rTMS, there was a significant difference and improvement in the severity of the clinical symptoms for ASD except for the level of activity and listening response and use of the body by comparing the severity of symptoms before and after rTMS. Comparing results before and after rTMS by the level of clinical severity of autism according to DSM-5; at the level of severity in social communication, the improvement was statistically significant ( $p$-value 0.001). At the level of severity in restricted and repetitive behaviors the improvement was statistically highly significant (p-value $<0.001)$. Conclusion: this study concluded that rTMS over left dorso-lateral prefrontal cortex may be safe and effective way of providing a relief of ASD symptoms.

Keywords: ASD - neurobiology - TMS - rTMS.

\section{INTRODUCTION}

Autism spectrum disorder (ASD) is one of the most common child psychiatric disorders, with a prevalence estimated at $1.1 \%$ of the population (1). Children diagnosed with ASD differ from typically developing children on many cognitive and behavioral dimensions, and therefore the term 'spectrum' is used to emphasize its full scope. The spectrum consists of a heterogeneous group of disorders, including Autism, Asperger's Syndrome, Pervasive Developmental Disorder- Not otherwise specified (PDD-NOS), Childhood Disintegrative Disorder, and Rett syndrome (2). ASD significantly impairs social interactions. For example, individuals with ASD are often unable to understand and interpret nonverbal behaviors in others, which can result in a failure to develop peer relationships ${ }^{(3)}$.

The diagnosis of ASD is based on observations and assessments of behavior using Diagnostic and Statistical Manual of Mental Disorders (DSM) or International Classification of Diseases (ICD) criteria. However, postmortem, genetic and neuroimaging data indicate that the behavioral ASD phenotype is the product of atypical brain development ${ }^{(4)}$. Several twin studies demonstrated that ASD is heritable. Monozygotic twins have a higher concordance rate than dizygotic twins, $90 \%$ and $10 \%$, respectively (5). However, the exact etiology is unknown, and it is likely that a combination of multiple genetic and environmental factors could result in ASD ${ }^{(6)}$.

Autism spectrum disorder is a complex neurodevelopmental disorder, which is accompanied by differences in brain anatomy, functioning and brain connectivity. Due to its neurodevelopmental character, and the large phenotypic heterogeneity among individuals on the autism spectrum, the neurobiology of autism spectrum disorder is inherently difficult to describe. Nevertheless, significant progress has been made in characterizing the neuroanatomical underpinnings of autism spectrum disorder across the human life span and in identifying the molecular pathways that may be affected in autism spectrum disorder. Moreover, novel methodological frameworks for analyzing neuroimaging data are emerging that make it possible to characterize the neuroanatomy of autism spectrum disorder on the case level and to stratify individuals based on their individual phenotypic make up $^{(7)}$.

The individual components of the neural systems underlying ASD are well established and include: (1) Fronto-temporal and fronto-parietal regions such as the medial, orbitofrontal (OFC) and inferior-frontal (IFG) cortices, the posterior parietal cortex, the superior temporal sulcus (STS) and the fusiform gyrus. (2) Limbic brain regions such as the amygdalahippocampal complex, the thalamus and cingulate regions. (3) The fronto-striatal circuitry including parts of the basal ganglia, the anterior cingulate cortex (ACC) and the dorsolateral prefrontal cortex (DLPFC). (4) The cerebellum ${ }^{(8)}$.

Moreover, many of the neural structures that have been reported as atypical in ASD overlap with the set of brain regions that are integral parts of the so-called 'social' and 'emotional' brain, which encompasses a set of brain regions involved in wider aspects of social cognition and emotional processing. For example, brain 
regions that are involved in social cognition include the medial prefrontal cortex (mPFC), the anterior cingulate cortex (ACC), the inferior frontal gyrus (IFG), the superior temporal sulcus (STS), the amygdala, and the anterior insula ${ }^{(9)}$. Thus, despite a long history of research on ASD, no much is known yet about the biological causes and how the disorder can be treated. This is partly due to the heterogeneity of the disease and the diversity of observed symptoms. At present, there is no cure for the core symptoms of ASD.

Treatment strategies, such as behavioral interventions and pharmacological treatments, all aimed to reduce symptoms. Pharmacological treatments are effective in treating comorbid features of ASD, such as catatonia and depression, but currently there is no pharmacotherapy that has shown to be effective in treating the core symptoms of ASD ${ }^{(10)}$. Furthermore, treatments such as behavioral and pharmacological interventions are nonspecific. To date, there are no treatment strategies that aim at a specific symptom or specific dysfunctioning brain area. Several studies have suggested that social skill deficits might lie at the heart of other symptoms in ASD ${ }^{(11)}$.

Over the past quarter century, neuroscience techniques have been developed and applied to ASD to study brain structure and function. Additionally, clinical trials of therapeutic interventions aimed at modulating brain functioning, have also been evaluated. In this article, we discussed one neuroscientific technique, namely transcranial magnetic stimulation (TMS) that has been used both to study the neural mechanisms of ASD as well as to therapeutically target the predicted dysfunction. TMS is a method for noninvasive focal brain stimulation, where localized intracranial electrical currents, large enough to depolarize a small population of neurons that are generated by rapidly changing extracranial magnetic fields ${ }^{(12)}$.

rTMS protocols involve the clinical treatment of neuropsychiatric disorders such as migraines, strokes, Parkinson's disease and psychiatric conditions, mainly depression ${ }^{(13)}$.

In addition to improving specific symptoms of depression and schizophrenia, rTMS has also been suggested to enhance cognition in neuropsychiatric disorders ${ }^{(14)}$. Observed beneficial effect on cognitive function, specifically on delayed recall, and a trend towards improvement on executive function, measured by the Stroop test. Interestingly, a study of inhibitory rTMS over the bilateral DLPFC on patients with high functioning ASD has also shown improvement in executive functioning, specifically in error monitoring and correction ${ }^{(15)}$.

Furthermore, a very recent double-blind controlled study reported that bilateral deep rTMS over the DLPFC resulted in a significant improvement of social relatedness in adults with ASD ${ }^{(16)}$. Results of published studies are promising suggesting that specific rTMS protocols targeting specific regions of cortex may lead to improvement in specific behavioral deficits in some individuals with ASD. And with this and other studies we may offer new hope to patients, researchers and clinicians in the treatment of ASD.

\section{AIM OF THE WORK}

To study the effect of repetitive transcranial magnetic stimulation on a sample of children with autism spectrum disorder who attended to Al-Azhar University Specialized Hospital, in the period from January 2019 to June 2019.

\section{PATIENTS AND METHODS}

This prospective study included 30 children that were chosen randomly from Psychiatry Clinic, Bab Al Shearia Al-Azhar University Hospital, in the period from January 2019 to June 2019 seeking for medical help and complaining from some behavioral and study problems.

Their ages ranged from 4 to 10 years old. After being diagnosed clinically according to DSM-5 through a designed semi-structured interview and through application of childhood autistic rating scale (CARS) ${ }^{(17)}$ as well as assessment of the degree of clinical severity of autism spectrum disorders according to DSM-5. Females were 5 children with percentage of $16.7 \%$, while males were 25 children with percentage of $83.3 \%$. All patients on the sample didn't stop their medical or behavioral therapy for ASD.

\section{Ethical and approval considerations:}

Oral and written consents were taken from parents of children taking into considerations maintaining the confidentiality of the data, consenting to visual footage, publications and most importantly acknowledgement of the potential side effects. In addition, approval of the Ethical Committee of Psychiatry Department in Bab Al Shearia hospital, Al-Azhar University also was taken to do the research.

\section{Included children were subjected to 3 stages: The first stage:}

a- Taking a full medical history - general medical examination.

b- Full psychiatric history in order to pick up and diagnose children with ASD clinically according to DSM-5 through a designed semi structured interview.

c- Application of Child Autism Rating Scale (CARS).

d- An assessment of the degree of clinical severity of autism spectrum disorders (American Psychiatric Association 2013), clinician-rated severity of autism spectrum and social communication disorders.

\section{The second stage:}

Application of transcranial magnetic stimulation: They received rTMS over the left dorso-lateral prefrontal cortex (DLPFC) for 6 sessions then over the right dorso-lateral prefrontal cortex for 6 sessions, at low frequency $1 \mathrm{~Hz}$ and intensity $90 \%$ of motor 
threshold ( 15 trains x $10 \mathrm{sec}, 150$ pulse per session at 26 sec interval) every week for total 12 consecutive weeks.

\section{The third stage :}

Re-application of the CARS to patients at the end of sessions, reassessment of the degree of clinical severity of autism spectrum disorder clinical evaluation and the results are tabulated and processed statistically.

\section{Inclusion criteria included the following:}

The age range was 4-10 years old, both sexes were included and who were diagnosed as ASD patients by the previously mentioned tools.

\section{Exclusion criteria included the following:}

Children with epilepsy, past or family history of seizures, children with history of brain lesions (post traumatic or any pathology) who may have a lower seizure threshold, children with any other psychiatric or neurological disease, children with intracranial metallic or magnetic pieces and children with implanted medication pump, intra-cardiac line or sever cardiac disease.

\section{Statistical analysis}

All data were collected and analyzed using SPSS program version 15.0 using t-test.

$\mathrm{P}$-value is considered significant $<0.05$.

\section{RESULTS}

Table (1): Comparison between results of CARS before and after conducting transcranial magnetic stimulation for 12 sessions:

\begin{tabular}{|c|c|c|c|c|c|c|}
\hline \multirow{2}{*}{\multicolumn{2}{|c|}{ CARS Test }} & \multicolumn{2}{|c|}{ After rTMS } & \multicolumn{2}{|c|}{ Before rTMS } & \multirow{2}{*}{ p-value } \\
\hline & & \multirow{2}{*}{$\frac{N=30}{9}$} & \multirow{2}{*}{$\frac{\%}{30.0}$} & \multirow{2}{*}{$\begin{array}{c}\mathrm{N}=30 \\
0\end{array}$} & \multirow{2}{*}{$\begin{array}{c}\% \\
0.0\end{array}$} & \\
\hline \multirow{4}{*}{ Relating to people } & Normal & & & & & \multirow{4}{*}{$<0.001 *$} \\
\hline & Mild & 19 & 63.3 & 11 & 36.7 & \\
\hline & Moderate & 2 & 6.7 & 16 & 53.3 & \\
\hline & Sever & 0 & 0.0 & 3 & 10.0 & \\
\hline \multirow{3}{*}{ Imitation } & Normal & 20 & 66.7 & 8 & 26.7 & \multirow{3}{*}{$0.006 * *$} \\
\hline & Mild & 8 & 26.7 & 20 & 66.7 & \\
\hline & Moderate & 2 & 6.7 & 2 & 6.7 & \\
\hline \multirow{4}{*}{ Emotional response } & Normal & 7 & 23.3 & 0 & 0.0 & \multirow{4}{*}{$0.001 * *$} \\
\hline & Mild & 20 & 66.7 & 14 & 46.7 & \\
\hline & Moderate & 3 & 10.0 & 13 & 43.3 & \\
\hline & Sever & 0 & 0.0 & 3 & 10.0 & \\
\hline \multirow{4}{*}{ Body use } & normal & 3 & 10.0 & 0 & 0.0 & \multirow{4}{*}{0.104} \\
\hline & Mild & 24 & 80.0 & 23 & 76.7 & \\
\hline & Moderate & 3 & 10.0 & 4 & 13.3 & \\
\hline & Sever & 0 & 0.0 & 3 & 10.0 & \\
\hline \multirow{4}{*}{ Object use } & normal & 7 & 23.3 & 4 & 13.3 & \multirow{4}{*}{$0.001 * *$} \\
\hline & Mild & 18 & 60.0 & 6 & 20.0 & \\
\hline & Moderate & 5 & 16.7 & 15 & 50.0 & \\
\hline & Sever & 0 & 0.0 & 5 & 16.7 & \\
\hline \multirow{4}{*}{ Adaptation to change } & normal & 10 & 33.3 & 12 & 40.0 & \multirow{4}{*}{$0.009 * *$} \\
\hline & Mild & 20 & 66.7 & 10 & 33.3 & \\
\hline & Moderate & 0 & 0.0 & 6 & 20.0 & \\
\hline & Sever & 0 & 0.0 & 2 & 6.7 & \\
\hline \multirow{4}{*}{ Visual response } & normal & 10 & 33.3 & 4 & 13.3 & \multirow{4}{*}{$0.001 * *$} \\
\hline & Mild & 14 & 46.7 & 5 & 16.7 & \\
\hline & Moderate & 6 & 20.0 & 14 & 46.7 & \\
\hline & Sever & 0 & 0.0 & 7 & 23.3 & \\
\hline \multirow{3}{*}{ Listening response } & normal & 8 & 26.7 & 4 & 13.3 & \multirow{3}{*}{0.126} \\
\hline & Mild & 20 & 66.7 & 19 & 63.3 & \\
\hline & Moderate & 2 & 6.7 & 7 & 23.3 & \\
\hline \multirow{4}{*}{$\begin{array}{l}\text { Taste, smell and touch } \\
\text { response and use }\end{array}$} & normal & 13 & 43.3 & 0 & 0.0 & \multirow{4}{*}{$<0.001 *$} \\
\hline & Mild & 15 & 50.0 & 16 & 53.3 & \\
\hline & Moderate & 2 & 6.7 & 11 & 36.7 & \\
\hline & Severe & 0 & 0.0 & 3 & 10.0 & \\
\hline & normal & 6 & 20.0 & 0 & 0.0 & \\
\hline Fear or nervoucnecs & Mild & 19 & 63.3 & 14 & 46.7 & $0005 * *$ \\
\hline Fear or nervousness & Moderate & 5 & 16.7 & 14 & 46.7 & $0.005^{n+4}$ \\
\hline & Severe & 0 & 0.0 & 2 & 6.7 & \\
\hline
\end{tabular}




\begin{tabular}{|c|c|c|c|c|c|c|}
\hline \multirow{2}{*}{\multicolumn{2}{|c|}{ CARS Test }} & \multicolumn{2}{|c|}{ After rTMS } & \multicolumn{2}{|c|}{ Before rTMS } & \multirow{2}{*}{ p-value } \\
\hline & & \multirow{2}{*}{$\frac{N=30}{2}$} & \multirow{2}{*}{$\%$} & \multirow{2}{*}{$\mathrm{N}=30$} & \multirow{2}{*}{$\begin{array}{c}\% \\
13.3\end{array}$} & \\
\hline \multirow{4}{*}{ Verbal communication } & normal & & & & & \multirow{4}{*}{$<0.001 *$} \\
\hline & Mild & 15 & 50.0 & 2 & 6.7 & \\
\hline & Moderate & 13 & 43.3 & 6 & 20.0 & \\
\hline & Severe & 0 & 0.0 & 18 & 60.0 & \\
\hline \multirow{4}{*}{$\begin{array}{l}\text { Non-Verbal } \\
\text { communication }\end{array}$} & normal & 2 & 6.7 & 2 & 6.7 & \multirow{4}{*}{$0.047 * *$} \\
\hline & Mild & 25 & 83.3 & 16 & 53.3 & \\
\hline & Moderate & 3 & 10.0 & 9 & 30.0 & \\
\hline & Severe & 0 & 0.0 & 3 & 10.0 & \\
\hline \multirow{4}{*}{ Activity level } & normal & 4 & 13.3 & 5 & 16.7 & \multirow{4}{*}{0.349} \\
\hline & Mild & 11 & 36.7 & 5 & 16.7 & \\
\hline & Moderate & 11 & 36.7 & 16 & 53.3 & \\
\hline & Severe & 4 & 13.3 & 4 & 13.3 & \\
\hline \multirow{4}{*}{$\begin{array}{l}\text { Level and consistency of } \\
\text { intellectual response }\end{array}$} & normal & 8 & 26.7 & 4 & 13.3 & \multirow{4}{*}{$0.013 * *$} \\
\hline & Mild & 14 & 46.7 & 7 & 23.3 & \\
\hline & Moderate & 8 & 26.7 & 13 & 43.3 & \\
\hline & Severe & 0 & 0.0 & 6 & 20.0 & \\
\hline \multirow{3}{*}{ General impressions } & normal & 13 & 43.3 & 4 & 13.3 & \multirow{3}{*}{$0.009 * *$} \\
\hline & Mild & 17 & 56.7 & 22 & 73.3 & \\
\hline & Moderate & 0 & 0.0 & 4 & 13.3 & \\
\hline \multirow[b]{2}{*}{ Total CARS score } & Mean & \multicolumn{2}{|c|}{31.4} & \multicolumn{2}{|c|}{40.2} & \multirow{2}{*}{$<0.001 *$} \\
\hline & $\begin{array}{l} \pm \text { Standard } \\
\text { deviation }\end{array}$ & \multicolumn{2}{|c|}{2.5} & \multicolumn{2}{|c|}{3.7} & \\
\hline
\end{tabular}

$*$ : p-value $<0.001$ is considered highly significant.

$* *$ : p-value $<0.05$ is considered significant.

The results of the study after the completion of 12 sessions of the rTMS showed a significant difference and improvement in the severity of the clinical symptoms for ASD except for the level of activity and listening response and use of the body by comparing the severity of symptoms before and after rTMS. By conducting statistical tests, the average measures of problems with relationship with people, sensory responses and verbal communication after sessions were relatively reduced. This relative decrease was found to be highly significant.The average measurements of problems: imitation, emotional response, object use and visual response, fear or nervousness, nonverbal communication, level of consistency of intellectual response, general impression as well as the total CARS score after conducting rTMS relatively decreased and found to be statistically significant. The average score in the CARS scale changed from 40.2 to 31.4 .

\section{DISCUSSION}

The current study aimed to study the potential therapeutic effect of rTMS in 30 patients diagnosed with ASD. It was hypothesized that using rTMS on some brain areas, namely this study selected dorso lateral prefrontal cortex (DLPFC) might improve core symptoms of autism spectrum disorder (ASD). In the current study, the ages of participating children $(\mathrm{N}=30)$ ranged from 4-10 years old with a mean age of 5.33 \pm $1.15 \mathrm{y}$. The sample as a whole was relatively young. This is unlike other studies in choosing the participants older in age. Most studies published till 2016, participants were older in age than current study, ranged between 13$33^{(18)}$.

In the current study, choosing participants younger in age that early intervention refers to brain plasticity theory and attempts to intervene earlier has better response than intervening later in life age ${ }^{\left({ }^{(19)}\right.}$. In other similar studies age of participants ranged between 12-27 years ${ }^{(20)}$. In Baruth et al. ${ }^{(21)}$, age ranged between 9-27 years and in Sokhadze et al. ${ }^{(22)}$, age ranged from 10 to 21 years old. Using narrower age range, in the current study made the group homogenous and thus alleviating the age factor difference that may contribute to different response to rTMS.

The DLPFC was chosen due to its extensive network connections with other specialized distributed and local networks in the brain which is not specific to one side ${ }^{(23)}$ and thus current study targeted both left and right DLPFC similar to Baruth et al. (21), Casanova et al. ${ }^{(23)}$ and Sokhadze et al. ${ }^{(24)}$ while Sokhadze et al. ${ }^{(25)}$ targeted left DLPC only.

Selecting $1 \mathrm{~Hz}$ as the stimulation frequency as studies have shown that low-frequency rTMS $(\leq 1 \mathrm{~Hz})$ increases inhibition of stimulated cortex ${ }^{(26)}$. There is also a lower risk for seizures with the lower the rTMS frequency. Like other similar studies, the current study used inhibitory $<1 \mathrm{~Hz}$ frequency $(21,24 \& 25)$. It has been suggested that the excitation / inhibition imbalance could be the key determinant in neuroplasticity abnormalities in neuro developmental disorders such 
$\mathrm{ASD}^{(27)}$ and a deficit in inhibitory neurotransmission has been implicated in the pathogenesis of the ASD. It is believed that such deficit could develop during neuronal maturation ${ }^{(28)}$. The selection of $90 \%$ of motor threshold (MT) was based on prior studies where rTMS was used for prefrontal stimulation in different psychiatric and neurological disorders ${ }^{(29,30)}$.

The current study answered the question aimed to study whether rTMS has potential therapeutic effect or not effective in treatment core symptoms of ASD. The results of the study after the completion of 12 sessions of the rTMS, comparing the severity of symptoms before and after rTMS, showed that there was a significant difference and improvement in the severity of the clinical symptoms for ASD except for the level of activity, listening response and use of the body. By conducting statistical tests, the average measures of problems with relating to people, sensory responses (Taste, smell and touch response and use) and verbal communication after sessions were relatively reduced. This relative decrease was found to be highly significant ( $p$ value $<0.001$ ). The average measurements of the problems of imitation, emotional response, object use, visual response, fear or nervousness, nonverbal communication, level of consistency of intellectual response and general impression as well as the total CARS score after conducting rTMS relatively decreased and it has been found to be statistically significant ( $\mathrm{p}$ value $<0.005$ ). The average score in the CARS scale changed from 40.2 to 31.4 .

Casanova et al. ${ }^{(23)}$ used similar TMS protocol typical to our study and showed that there was a significant difference between groups in reduction of repetitive and restricted behavior patterns following 12 sessions of bilateral rTMS as measured by the repetitive behavior scale (RBS). There was also a statistically significant group differences in reduction in irritability as measured by the aberrant behavior checklist (ABC). No changes in hyperactivity reached significance as a result of rTMS. No changes in social awareness (unlike our study). They enrolled 45 autistic patients with age ranged from 9 to 19 years. Forty-one participants were high-functioning persons with autism diagnosis and 4 had Asperger Syndrome with less severe symptomatology than in our sample. They included low functioning subjects with significant behavioral problems and social. In other words, the high functioning autism subjects in Casanova et al. ${ }^{(24)}$ study did not have many problems, so there was no much change following rTMS unlike that in our study. It is supposed that using TMS in younger age (4-10 years) in our study is a reason for better response than in other studies.

Sokhadze et al. (25) showed similar results following rTMS. Subjects were reported to have reduced repetitive-ritualistic behavior as measured by the Repetitive Behavior Scale, but no changes in social awareness and irritability, or hyperactivity were observed. The TMS treatment course was administered two times per week for 3 weeks (a total of six $0.5 \mathrm{~Hz}$ rTMS treatments, 150 pulse per session) over the left DLPFC only. In the current study, the used rTMS protocol was more extensive of weekly sessions for 12 weeks with the 1st six treatments over the left DLPFC, whereas the remaining six treatments were over the right DLPFC. TMS was administered inhibitory at higher 1.0 $\mathrm{Hz}$ frequency and $90 \%$ of MT, total of 150 pulses per day session with 10 trains with 15 pulses each, 20-30 seconds intervals between the trains. Better results were seen in Sokhadze et al. ${ }^{(25)}$ than that were in our study.

A study similar to our study rTMS protocol but with completing 18 sessions rather than 12 sessions in our study, Sokhadze et al. ${ }^{(22)}$ administered 18 sessions of $1 \mathrm{~Hz}$ rTMS applied over the Dorso-Lateral Prefrontal cortex in 27 individuals with ASD diagnosis. The waiting list group comprised 27 age-matched subjects with ASD tested twice. Both TMS and waiting list groups were assessed at the baseline and after completion of 18 weekly sessions of rTMS (or wait period). Post-TMS evaluations showed decreased irritability and hyperactivity on the Aberrant Behavior Checklist (ABC), and decreased stereotypic behaviors on the Repetitive Behavior Scale (RBS-R). The study indicated that rTMS improves executive functioning in ASD as evidenced by normalization of ERP responses and behavioral reactions (RT Reaction Time accuracy) during executive function test ${ }^{\text {(22) }}$.

None of the children in the sample experienced significant side effects during the study.

\section{CONCLUSION}

This study concluded that repeated sessions of rTMS over left dorso-lateral prefrontal cortex has the potential to become an important therapeutic tool in ASD treatment and has shown significant benefits in treating core symptoms of ASD.

\section{REFERENCES}

1. Centers for Disease Control and Prevention (2012): Prevalence of Autism Spectrum Disorders - Autism and Developmental Disabilities Monitoring Network, 14 Sites, United States, 2008. Morbidity and Mortality Weekly Report, 61: 3-11.

2. American Psychiatric Association (2000): Diagnostic and Statistical Manual of Mental Disorders, Fourth Edition, Text Revision. Washington, DC, American Psychiatric Association. https://behavenet.com/diagnostic-and-statistical-manualmental-disorders-fourth-edition-text-revision.

3. Cotugno AJ (2009): Social competence and social skills training and intervention for children with autism spectrum disorders. Journal of Autism and Developmental Disorders, 39: 1268-1277.

4. Ameis SH, Catani M (2015): Altered white matter connectivity as a neural substrate for social impairment in Autism Spectrum Disorder. Cortex, 62: 158-181. 
5. Faras H, Al Ateeqi N, Tidmarsh L (2010): Autism spectrum disorders. Annals of Saudi Medicine, 30: 295300.

6. Oberman LM, Rotenberg A, Pascual-Leone A (2013): Use of transcranial magnetic stimulation in autism spectrum disorders. Journal of Autism and Developmental Disorders, 43: 1-13.

7. Ecker C (2017): The neuroanatomy of autism spectrum disorder: An overview of structural neuroimaging findings and their translatability to the clinical setting. Autism: The International Journal of Research and Practice, 21 (1): 18-28.

8. Amaral DG, Schumann CM, Nordahl CW (2008): Neuroanatomy of autism. Trends in Neurosciences, 31 (3): 137-145.

9. Blakemore SJ (2008): The social brain in adolescence. Nature Reviews. Neuroscience, 9 (4): 267-277.

10. Oberman LM (2012): mGluR antagonists and GABA agonists as novel pharmacological agents for the treatment of autism spectrum disorders. Expert Opinion on Investigational Drugs, 21: 1819-1825.

11. Gutstein SE, Whitney T (2002): Asperger syndrome and the development of social competence. Focus on Autism and Other Developmental Disabilities, 17: 161-171.

12. Wagner T, Valero-Cabre A, Pascual-Leone A (2007): Noninvasive human brain stimulation. Annu Rev Biomed Eng., 9: 527-565.

13. Croarkin PE, Wall CA, Lee J (2011): Applications of transcranial magnetic stimulation (TMS) in child and adolescent psychiatry. International Reviews of Psychiatry, 23: 445-453.

14. Demirtas-Tatlidede A, Vahabzadeh-Hagh AM, Pascual-Leone A (2013): Can noninvasive brain stimulation enhance cognition in neuropsychiatric disorders? Neuropharmacology, 64: 566-578.

15. Sokhadze EM, Baruth JM, Sears L, Sokhadze GE, ElBaz AS, Casanova MF (2012): Prefrontal neuromodulation using rTMS improves error monitoring and correction function in autism. Applied Psychophysiology and Bio Feedback, 37: 91-102.

16. Enticott PG, Firzgibbon BM, Kennedy HA, Arnold SL, Elliot D, Peachey A, Zangen A, Fitzgerald PB (2014): A double blind, randomized trial of deep repetitive transcranial magnetic stimulation (rTMS) for autism spectrum disorder. Brain Stimul., 7 (2): 206-11.

17. Schopler E, Reichler RJ, DeVellis RF, Daly K (1980): Toward objective classification of childhood autism: Childhood Autism Rating Scale (CARS). Journal of Autism and Developmental Disorders, 10 (1): 91-103.

18. Oberman L, Rotenberg A, Pascual-Leone A (2015): Use of transcranial magnetic stimulation in autism spectrum disorders. Journal of Autism Development Disorder, 45 (2): 524-536.

19. Geoffray MM, Thevenet $M$, Georgieff $\mathbf{N}$ (2016): News in early intervention in autism. Psychiatria Danubina., 28: $66-70$.
20. Sokhadze EM, El-Baz A, Baruth J, Mathai G, Sears L, Casanova MF (2009): Effects of low frequency repetitive transcranial magnetic stimulation (rTMS) on gamma frequency oscillations and event-related potentials during processing of illusory figures in autism. Journal of Autism and Developmental Disorders, 39 (4): 619-634.

21. Baruth JM, Casanova MF, El-Baz A, Horrell T, Mathai G, Sears L, Sokhadze E (2010): LowFrequency Repetitive Transcranial Magnetic Stimulation (rTMS) Modulates Evoked-Gamma Frequency Oscillations in Autism Spectrum Disorder (ASD). Journal of Neurotherapy, 14 (3): 179-194.

22. Sokhadze EM, El-Baz AS, Tasman A, Sears LL, Wang Y, Lamina EV, Casanova MF (2014): Neuromodulation integrating rTMS and neurofeedback for the treatment of autism spectrum disorder: an exploratory study. Applied Psychophysiology and Biofeedback, 39 (3-4): 237-257.

23. Casanova MF, Sokhade E, Opris I, WangY, Li X (2015): Autism Spectrum disorders: lonking neuropathological findings to treatment with trans magnetic stimulation. Acta Pediatrica, 104 (4): 346-55.

24. Casanova MF, Baruth JM, El-Baz A, Tasman A, Sears L, Sokhadze E (2012): Repetitive Transcranial Magnetic Stimulation (rTMS) Modulates Event-Related Potential (ERP) Indices of Attention in Autism. Translational Neuroscience, 3 (2): 170-180.

25. Sokhadze E, Baruth J, Tasman A, Mansoor M, Ramaswamy R, Sears L, Casanova MF (2010): Lowfrequency repetitive transcranial magnetic stimulation (rTMS) affects event-related potential measures of novelty processing in autism. Applied Psychophysiology and Biofeedback, 35 (2): 147-161.

26. Maeda F, Keenan JP, Tormos JM, Topka H, PascualLeone A (2000): Modulation of corticospinal excitability by repetitive transcranial magnetic stimulation. Clin Neurophysiol., 111: 800-805.

27. Rajji TK, Rogasch NC Daskalakis ZJ, Fitzgerald PB (2013): Neuroplasticity -based brain stimulation interventions in the study and treatment of schizophrenia: a review. Can J Psychiatry, 58 (2): 93-8.

28. Baroncelli L, Braschi C, Spolidoro M, Begeneisic T, MaffeibL, Sale A (2011): Brain plasticity and disease: a matter of inhibition. Neural Plast. https://www.hindawi.com/journals/np/2011/286073/abs/

29. Loo CK, Mitchell PB (2005): A review of the efficacy of transcranial magnetic stimulation (TMS) treatment for depression, and current and future strategies to optimize efficacy. Journal of Effective Disorders, 88 (3): 255-267.

30. Greenberg BD (2007): Transcranial magnetic stimulation in anxiety disorders. In: George MS, Belmaker RH, editors. Thanscranial magnetic stimulation in clinical psychiatry. Washington, DC: American Psychiatric Publishing Inc. 